\title{
Corneal crosslinking as a new therapeutic tool
}

\begin{abstract}
Corneal collagen cross-linking (CXL) is the most prevalent therapeutic approach for progressive Keratoconus. However, literature suggests that cross linking alone or in combination with other techniques has a beneficial impact on a series of corneal related diseases and states. This article attempts to provide a contemporary review for all additional applications of CXL. Specifically, it punctuates the latest studies that demonstrate the beneficial impact of CXL for iatrogenic ectasias, pellucid marginal degeneration, infectious keratitis, bullous keratopathy, and for ulcerative keratitis. The outcome of this review indicates that CXL could serve as a primary or adjuvant therapeutic modality for all aforementioned corneal-related pathologic states.
\end{abstract}

Keywords: corneal collagen cross-linking, review, iatrogenic ectasia, pellucid marginal degeneration, bullous keratopathy, infectious keratitis, intracorneal ring segment (ICRS), laser-assisted in situ keratomileusis (LASIK), photorefractive keratectomy (PRK)
Volume I Issue 3 - 2014

\author{
Vassilios Kozobolis, Haris Sideroudi, Georgios \\ Labiris \\ Eye Institute of Thrace EIT, Democritus University, Greece
}

Correspondence: : Vassilios Kozobolis, Professor of

Ophthalmology, Eye Institute of Thrace EIT, Democritus University of Thrace, Alexandroupolis, Greece, Tel 255I03099I,

Email kozobolis@gmail.com

Received: September 03, 2014 | Published: October 17, 2014
Abbreviations: CXL, corneal crosslinking; PACK-CXL, photo-activated chromophore for infectious keratitis crosslinking; ICRS, intracorneal ring segment; LASIK, laser-assisted in situ keratomileusis; PRK, photorefractive keratectomy; UCVA, uncorrected visual acuity; BCVA, best corrected visual acuity; UV-A, ultraviolet-A

\section{Introduction}

Corneal collagen cross linking is a minimally invasive technique that combines riboflavin and Ultraviolet-A (UV-A) irradiation for the production of a stiffening effect capable of augmenting the rigidity of the corneal tissue. CXL attempts to stabilize and strengthen the ectatic cornea by creating new covalent bonds between the stromal collagen fibrils, quasi "freezing" the cornea. The most common use of CXL is the management of ectatic disorders by halting their progression. Nevertheless, a continuous line of new applications is under investigation with promising results.

\section{Discussion}

\section{CXL in resistant ulcers}

CXL has been used recently in treatment of resistant corneal ulcer. This use of CXL technology is called Photo-Activated Chromophore for infectious Keratitis cross-linking (PACK-CXL). Hafezi et al., ${ }^{1}$ in a recent, randomized, prospective clinical trial involved 40 patients with advanced infectious keratitis and corneal melting. ${ }^{1}$ Twenty-one eyes were treated with both antimicrobial therapy and PACK-CXL and 19 control eyes received only antimicrobial therapy. The size of the corneal ulcers was significantly larger in the PACK-CXL group and the duration to the complete healing shorter.

\section{CXL in bullous keratopathy}

CXL has been shown to have an antiedematous effect on the cornea. Kozobolis et al. evaluated the therapeutic effect of corneal CXL bullous keratopathy combined with corneal ulcer. ${ }^{2}$ Within 24 hours of the treatment, patients reported significant subjective improvement of their visual acuity and ocular discomfort. Clinical evaluation revealed improvement of the corneal ulcer and the bullous keratopathy associated with significant decrease of the corneal thickness and haziness. During the 2-month follow-up period, a significant improvement of visual acuity was recorded. Despite the encouraging results, larger amount of patients and longer followup is necessary to confirm the long-term impact of CXL in bullous keratopathy.

\section{Correcting refractive error}

CXL is also being investigated as a nonsurgical method for correcting refractive error. New technology and devices are in market which can be used to deliver user-specified CXL patterns, including patterns designed to alter the patient's refraction. Most recent Kanellopoulos et al proposed novel application of toric topographically customized trans epithelial CXL aiming to achieve refractive astigmatic changes in a keratoconic cornea. ${ }^{3}$ They administered a specially formulated riboflavin transepithelial and applied a highfluence UV-A in a topographically customized pattern in an eye with progressive keratoconus. They introduce herein the novel application of a topographically customizable transepithelial CXL in progressive keratoconus in order to achieve an astigmatic refractive effect and ectasia stabilization. They proposed that aforementioned novel technique offers a non-ablative and non-incisional approach to treat irregular astigmatism in ectatic cornea with rapid visual rehabilitation. By placing that zone of flattening carefully, changing the shape of the zone and adjusting other parameters like the depth of the treatment and the dosing of the light, it is possible to customize the refractive result that achieved.

\section{CXL after refractive surgery}

LASIK is the most prevalent type of refractive surgery, however regardless of the important technical advances, certain limitation remain, such as the ectasias and the refractive regression. ${ }^{4-6}$ The initial presentation of post-LASIK ectasia can vary from 1 week up to several years. The symptoms are usually astigmatism and myopia, decrease in Uncorrected Visual Acuity (UCVA) and frequently in Best Corrected Visual Acuity (BCVA), as well. ${ }^{4}$ Nowadays there are many options for treatment ${ }^{5,7,8}$ however sometimes the corneal transplant is a necessity. ${ }^{7}$ Nowadays, many physicians combine refractive surgery and CXL in order to correct refractive errors in healthy eyes (LASIK plus CXL) or to treat keratoconus and ectasia (PRK plus CXL). LASIK in combination with CXL is used to improve refractive outcomes after LASIK in healthy eyes. CXL is used to stabilize the cornea, which may have become destabilized as a result corneal flap creation. ${ }^{9}$ Corneal biochemical response has been associated with the 
pathophysiology of regression after LASIK; therefore, modifying the biochemical properties of the cornea may result in positive treatment outcomes. ${ }^{6} \mathrm{CXL}$ in combination with LASIK drives in stable refractive correction over time and prevention of post-LASIK ectasia. ${ }^{4-6}$ Although current results are encouraging, this concept is still in a clinical investigational phase.

PRK in combination with CXL is used to improve the CXL process in eyes with keratoconus or ectasia. CXL is an effective tool against the progression of keratoconus, but the improvement in visual acuity is not always reported. Combination of two treatments seems to be a better approach in order to improve visual outcomes. ${ }^{10}$ Currently, topography-guided PRK followed by CXL seems to be the best combination for optimum results in the treatment of keratoconic patients. At this manner it is managed to offer functional vision and halt of the ectatic disorder. Further studies with longer follow-up are required to confirm the promising results of this methodology.

\section{CXL in combination with ICRS for the treatment of keratectasia}

Studies on ICRS implantation for the treatment of keratoconus have indicated more than two lines of improvement in visual acuity. ${ }^{11,12}$ A combination of ICRS with CXL treatment would, therefore exert a distinct beneficial impact on the ectatic cornea that aims to ${ }^{1}$ the improvement of corneal topography and visual ability $a^{2} d^{2}$ the stabilization of the ectatic cornea over time. In a retrospective case series, Chan et al. show that the addition of CXL to the ICRS procedure resulted in better outcomes than the ICRS insertion alone. ${ }^{13}$ Furthermore Coskunseven et al. suggested that ICRS implantation followed by the CXL procedure produced better results in $\mathrm{KC}$ than CXL followed by ICRS implantation. ${ }^{14}$ Moreover in the same concept Sideroudi et al reported that CXL procedure leads to the crosslinking effect not only of the corneal collagen but of the ICRS core material as well. This finding should be taken into consideration prior to any CXL treatment of post ICRS implanted cornea. ${ }^{15}$

\section{Conclusion}

CXL is a promising therapeutic intervention for corneal tissue rehabilitation in diseases that manifest with progressive keratectasia like keratoconus. In combination with refractive correction treatments (LASIK, PRK, ICRS), CXL provide advantageous results that each method alone. Moreover, CXL antiedematous and antimicrobial properties were demonstrated in a series of studies suggesting its therapeutic indications in bullous keratopathy and in infectious keratitis as an adjuvant treatment to conventional therapeutic modalities. ${ }^{1,2}$

\section{Acknowledgments}

None.

\section{Conflicts of interest}

Author declares that there is no conflict of interest.

\section{References}

1. Hafezi F, Randleman JB. PACK-CXL: defining CXL for infectious keratitis. J Refract Surg. 2014;30(7):438-439.

2. Kozobolis V, Labiris G, Gkika M, et al. UV-A collagen cross-linking treatment of bullous keratopathy combined with corneal ulcer. Cornea. 2010;29(2):235-238.

3. Kanellopoulos AJ, Dupps WJ, Seven I, et al. Toric topographically customized transepithelial, pulsed, very high-fluence, higher energy and higher riboflavin concentration collagen cross-linking in keratoconus. Case Rep Ophthalmol. 2014;5(2):172-180.

4. Celik HU, Alagoz N, Yildirim Y, et al. Accelerated corneal crosslinking concurrent with laser in situ keratomileusis. J Cataract Refract Surg. 2012;38(8):1424-1431.

5. Kanellopoulos AJ. Long-term safety and efficacy follow-up of prophylactic higher fluence collagen crosslinking in high myopic laserassisted in situ keratomileusis. Clin Ophthalmol. 2012;6:1125-1130.

6. Aslanides IM, Mukherjee AN. Adjuvant corneal crosslinking to prevent hyperopic LASIK regression. Clin Ophthalmol. 2013;7:637-641.

7. Binder PS, Lindstrom RL, Stulting RD, et al. Keratoconus and corneal ectasia after LASIK. J Cataract Refract Surg. 2005;31(11):2035-2038.

8. Bromley JG, Randleman JB. Treatment strategies for corneal ectasia. Curr Opin Ophthalmol. 2010;21(4):255-258.

9. Dupps WJ Jr, Wilson SE. Biomechanics and wound healing in the cornea. Exp Eye Res. 2006;83(4):709-720.

10. Labiris G, Giarmoukakis A, Sideroudi H, et al. Impact of keratoconus, cross-linking and cross-linking combined with photorefractive keratectomy on self-reported quality of life. Cornea. 2012;31(7):734-739.

11. Rabinowitz YS. Intacs for keratoconus. Curr Opin Ophthalmol. 2007;18(4):279-283.

12. Miranda D, Sartori M, Francesconi C, et al. Ferrara intrastromal corneal ring segments for severe keratoconus. J Refract Surg. 2003;19(6):645-653.

13. Chan CC, Sharma M, Wachler BS. Effect of implantation of inferiorsegment Intacs with and without C3-R on keratoconus. J Cataract Refract Surg. 2007;33(1):75-80.

14. Coskunseven E, Jankov MR, Hafezi F, et al. Effect of treatment sequence in combined intrastromal corneal rings and corneal collagen crosslinking for keratoconus. J Cataract Refract Surg. 2009;35(12):2084-2091.

15. Sideroudi H, Labiris G, Soto-Beobide A, et al. The effect of Collagen Cross-linking procedure on the material of Intracorneal Ring Segments. Curr Eye Res. 2014;15:1-6. 